\title{
Albedo heterogeneity on the surface of (1943) Anteros
}

\author{
Joseph Masiero ${ }^{1,2}$
}

\begin{abstract}
We have investigated the effect of rotation on the polarization of scattered light for the near-Earth asteroid (1943) Anteros using the Dual Beam Imaging Polarimeter on the University of Hawaii's $2.2 \mathrm{~m}$ telescope. Anteros is an L-type asteroid that has not been previously observed polarimetrically. We find weak but significant variations in the polarization of Anteros as a function of rotation, indicating albedo changes across the surface. Specifically, we find that Anteros has a background albedo of $p_{v}=0.18 \pm 0.02$ with a dark spot of $p_{v}<0.09$ covering $<2 \%$ of the surface.
\end{abstract}

\section{Introduction}

As the last remnants from an epoch of accretive formation, asteroids provide us windows into the composition and history of the inner Solar System. Except for the few largest bodies, asteroids did not heat up enough via decay of short-lived radionuclides or dissipation of gravitational potential energy to undergo complete differentiation. As such the minerals observed on their surfaces capture the elemental and temperature history of the local region of the protoplanetary disk at the time of their formation. By understanding asteroid surfaces we can directly probe those early disk conditions.

As the illuminated cross section of an asteroid changes the observed brightness fluctuates. Given a large enough sample of data a full shape model of a rotating body can be constructed (Kaasalainen, et al. 2001) even though it is unresolved. Photometric surveys for asteroid light curves have set limits on the composition and density of asteroids as a population (Pravec, et al. 2002) and have estimated the average shape distribution of small Main Belt asteroids (Masiero, et al. 2009a). All of these results however assume that the light curve is dominated by the object's shape and that the entire surface has a uniform composition and albedo.

It is possible to test for albedo variations using optical imaging polarimetry, color variations, or even simply photometric variations under the assumption of a regular shape (Akimov, et al. 1983). In the case of polarimetry there are strong empirical correlations between the albedo of an asteroid and both the slope of the polarization-phase curve and the location of the minimum

\footnotetext{
${ }^{1}$ Institute for Astronomy, University of Hawaii, 2680 Woodlawn Dr, Honolulu, HI 96822

${ }^{2}$ Jet Propulsion Lab, California Institute of Technology, 4800 Oak Grove Dr., MS 264-767, Pasadena, CA 91106, Joseph.Masiero@jpl.nasa.gov
} 
(negative) polarization (most recently: Cellino, et al. 1999). The polarization of light scattered off of an atmosphereless body as a function of phase angle depends on the distance between scattering elements and their index of refraction (Muinonen 1989; Muinonen, et al. 2002c). Index of refraction is an inherent mineralogical property and recent work has shown that the inter-element scattering distance is likewise determined by the surface chemistry (Masiero, et al. 2009b). It is not unexpected then that asteroids of different spectral classification show different polarization-phase curves (Muinonen, et al. 2002b), or that a differentiated-then-broken object like (4) Vesta would show polarization variations with rotation.

In almost every way investigated so far the asteroid Vesta stands out as an interesting and unique object, and this is similarly the case for albedo variation studies. Although a handful of other asteroids have weak detections of rotational modulation of their polarization and thus albedos (e.g. Broglia \& Manara 1992, etc.), Vesta represents one clear case of an object with polarization changes across its surface caused by changes in composition (Degewij. et al. 1979; Lupishko, et al. 1988; Broglia \& Manara 1989). This, along with photometry, spectroscopy and adaptive optic imaging, has lead to the current interpretation of Vesta as a differentiated body that has undergone a nearly-catastrophic impact event leaving a giant crater in its southern hemisphere. The crater reveals a now-solid mantle distinctly different in color and composition from the original crust material (Cellino, et al. 1987; Thomas, et al. 1997).

Nakavama, et al. (2000) found rotational modulation of the polarization for the asteroid (9) Metis with amplitude similar to what is observed for Vesta. Metis is a $D \sim 180 \mathrm{~km}$ asteroid that may have two large spots of significantly higher albedo $\left(p_{v} \sim 0.24-0.28\right)$ than the background material $\left(p_{v}=0.14\right)$. The authors find that both bright areas are on the leading (for prograde rotation) or trailing (for retrograde rotation) faces of the model ellipsoid (Mitchell, et al. 1995). The cause of albedo heterogeneity across the surface of objects smaller than Vesta is still undetermined. It is possible that non-disruptive collisions with impactors of different composition can leave localized deviations from the average mineralogy. Alternatively, a late formation with a history free of melting may preserve the varied composition of the protoplanetary disk. However this theory is complicated by recent work showing that asteroids likely were born big, and most objects smaller than a few hundred kilometers in diameter should be collisionally-created fragments (Morbidelli, et al. 2009).

Identifying albedo variations for small asteroids allows us to evaluate the accuracy of the assumption that flux changes are solely dependent on shape. This has important implications for results based on this assumption, especially shape models. Additionally we can also quantify the effect of collisions between small bodies in determining an asteroid's local regolith properties.

All asteroid polarization-phase curves follow the same general trend with increasing phase angle: zero polarization at zero phase, becoming negative to some minimum value and then increasing in an approximately linear fashion. Note that as is standard for Solar system polarimetry the reference direction for the angle of polarization is aligned with the vector perpendicular to the plane of scattering such that "positive" and "negative" polarization are defined as perpendicular 
and parallel to the scattering plane, respectively. The results presented here follow this convention. Each polarization-phase curve displays three distinguishing values used to classify its properties: the minimum negative polarization $\left(P_{\min }\right)$, the phase angle at which the polarization returns to zero (the inversion angle, $\alpha_{0}$ ) and the linear slope of the polarization-phase relation beyond the inversion angle $(h)$. Making use of the albedo-polarization relation from Cellino, et al. (1999),

$$
\log p_{v}=(-1.12 \pm 0.07) \log h-(1.78 \pm 0.06)
$$

(where $p_{v}$ is the geometric V-band albedo) we can use imaging polarimetry to test for changes in polarization that directly indicate albedo heterogeneity across an asteroid's surface.

\section{Observations and Discussion}

Changes in the polarization of the scattered light across the surface of an asteroid will be small even in the best-case scenarios. To obtain a significant measurement of the largest of these variations we require an instrument that can attain better than $0.1 \%$ polarization accuracy. Our study made use of the Dual Beam Imaging Polarimeter (DBIP) located on the University of Hawaii's $2.2 \mathrm{~m}$ telescope on Mauna Kea, Hawaii (Masiero, et al. 2007). DBIP uses a double-calcite Savart plate in series with a quarter-wave and a half-wave retarder to simultaneously measure linear and circular polarization with accuracy better than $0.1 \%$ (Masiero, et al. 2008). DBIP uses a $g^{\prime}+r^{\prime}$ filter with a bandpass of $400-700 \mathrm{~nm}$. While asteroid polarization does depend on color (Cellino et al. 2005) changes are usually small in this wavelength range and typically within measurement errors.

Observation of our target asteroid were supplemented with polarized and unpolarized standards to confirm consistency of setup, stability of the instrument, and accuracy of the measurements. Standards were taken from Fossati, et al. (2007) as well as the standard list for Keck/LRISp1 which includes the Hubble Space Telescope polarimetric standards (Schmidt, et al. 1992). These measurements all verified that the errors were within the range expected from previous calibrations.

As albedo is related to the polarization-phase slope $h$, for a given albedo variation the respective polarization change will be larger when observed at higher phase angles (for $\alpha>\alpha_{0}$ ). Geometric restrictions prevent Main Belt asteroids (MBAs) from ever reaching phases angles larger than $\alpha \sim 30^{\circ}$, but near-Earth asteroids (NEAs) pass closer to Earth and so can reach much larger phase angles. For this reason, NEAs are preferred targets when looking for albedo variations. At high phases the polarization of scattered light takes on a linear trend that increases up to the level of $\sim 5-10 \%$ polarized depending on surface mineralogy. These large polarizations mean that any variation with rotation at high phases can be easily interpreted as changes in the integrated surface albedo using the slope-albedo relation (Eq1) and that the absolute value for the range of the albedo can be determined.

\footnotetext{
${ }^{1}$ http://www2.keck.hawaii.edu/inst/lris/polarimeter/polarimeter.html
} 
From July to September of 2009 the NEA (1943) Anteros passed through phase angles of $16-40^{\circ}$ all while brighter than $V=17$ mag presenting a prime opportunity to measure the polarization, slope and albedo with high accuracy. The optical/NIR spectrum of Anteros displays a spectral slope comparable to S-types but with a muted $1 \mu \mathrm{m}$ absorption band resulting in a classification of L-type (Binzel, et al. 2004). With a measured period of $P=2.8695 \pm 0.0002 \mathrm{hr}$ and a single-peaked photometric light curve with amplitude $A=0.09$ mag (Pravec, et al. 1998), Anteros is an excellent target to test for rotational variation in polarization and albedo in a few nights of observing. In particular, a single-peaked low-amplitude light curve indicates a shape very close to spherical. (Pravec, et al. (1998) found an amplitude of 0.09 mag across phase angles ranging from $19^{\circ}<\alpha<32^{\circ}$, resulting in a shape approximation of $a / b<1.1$ ).

In Table 1 we present our polarimetric observations of Anteros. Included for each night are the $\mathrm{V}$ magnitude, exposure time, number of 6-exposure polarimetry measurements acquired $\left(n_{\text {meas }}\right)$, phase angle, ecliptic longitude, summed linear polarization of all measurements and linear polarization angle with respect to the vector perpendicular to the scattering plane $\left(\theta_{p}\right)$. No significant circular polarization was detected on any of the nights. The average nightly polarizations are shown in Fig 1 along with generic model polarization-phase curves for typical S-type asteroids (dotted) and C-type asteroids (dashed). The model curves were made using the linear-exponential modeling technique presented by Muinonen, et al. (2002a) and fitted by-eye to the data shown in Fig 1 of Muinonen, et al. (2002b), to act as useful approximations. The constants used in this case were, for the C-type model: $P_{a}=5.5, P_{d}=6, P_{k}=0.3$ and for the S-type model: $P_{a}=4.3, P_{d}=12$, $P_{k}=0.17$.

The polarization of Anteros is clearly most closely related to an S-type polarization curve as expected from its spectral features. We measure for Anteros an inversion angle of $\alpha_{0}=20.3 \pm 0.3^{\circ}$ and a slope beyond the inversion angle of $h=0.122 \pm 0.001$. Both $\alpha_{0}$ and $h$ (and their respective errors) were found by conducting a least-squares minimization fit of a line to the four nights of data. As the data span a large range of phases and have small individual errors, the resultant error on $h$ is small. Following Eq 1 we derive a bulk albedo of $p_{v}=0.175 \pm 0.002 \pm 0.02$ (errors relative and absolute, respectively). Note that the limiting error on albedo $\left(\sigma_{a b s}=0.02\right)$ derives from the uncertainty on the constants in Eq 1 and will affect absolute albedo measurements. This does not apply to relative comparisons between measured albedos, which have an error of $\sigma_{\text {rel }}=0.002$ in the above case. For all observations reported here, the calibration error on Eq11 is greater than the noise error by nearly an order of magnitude, and thus dominates the final error on the measured albedos. (All errors reported in this paper are $1 \sigma$.)

Following (Muinonen, et al. 2002c) polarization can be approximated as

$$
P \sim \frac{\alpha^{2}}{2 n}-\left(\frac{n-1}{n+1}\right)^{2} \frac{(k d \alpha)^{2}}{2\left[1+(k d \alpha)^{2}\right]}
$$


and solving this for the case of zero polarization at the inversion angle we find

$$
\alpha_{0} \sim \sqrt{n\left(\frac{n-1}{n+1}\right)^{2}-\frac{1}{(k d)^{2}}}
$$

where $k=2 \pi / \lambda, d$ is the inter-element scattering distance, and $n$ the index of refraction. For a central wavelength of $0.55 \mu \mathrm{m}$ for DBIP and a typical scattering distance for NEAs of $d \sim 4 \mu \mathrm{m}$ (Masiero, et al. 2009b) the second term on the right in Eq2 2 is negligible, and the index of refraction can be determined for a given inversion angle. For Anteros we find an index of refraction of $n \sim 1.74$.

In Fig 2 we show the nightly polarization light curves for Anteros. Observations have been wrapped to the measured $2.8695 \mathrm{hr}$ photometric period and all measurements within a 0.1 phase bin have been co-added to reduce measurement error. The zero point for rotation phase was chosen arbitrarily on the first night. When all nights of data are wrapped to this zero-point the error on the period translates to a phase error of \pm 0.01 rotations between each observing night. Thus, features at specific phases can be compared across nights.

We find weak variation in the polarization at a $4 \sigma$ significance level for the night of 2009-07-22 with an amplitude of $0.3 \%$ when comparing the data in the rotation phase range of $0.6-0.8$ to the data between rotation phases $0.8-0.4$. These polarization changes most likely indicate a variation in the albedo across the surface of Anteros. The amplitude of the polarization variation scales with the absolute polarization so it is not surprising that the other observing nights at lower phases show no clear variation, e.g. a variation with amplitude of $P=0.3 \%$ at a phase of $\alpha=40.5^{\circ}$ would be expected to have an amplitude of $P=0.1 \%$ at a phase of $\alpha=28.0^{\circ}$ which is below our threshold for significant detection. Additionally, changes in the observing geometry between observations could cause an area that was observable on the night of 2009-07-22 to have a reduced visibility on subsequent observations, or even be beyond the horizon. However even in the most extreme case, where the rotation axis is parallel the to plane of Earth's orbit, the line-of-sight vector only moves a total of $\sim 18^{\circ}$ with respect to the rotation axis over the dates of the observations (this is equivalent to the change in ecliptic longitude). Though this could account for the changes in polarization under specific circumstances, it is not the most likely scenario.

It has been suggested that the change in polarization alternatively could be due to an extreme topographical feature that deviates significantly from the surrounding area. At the high phases at which we observed Anteros first-order scattering is dominant and so we may simply apply basic scattering properties to the surface (e.g. the angle of incidence and angle of scattering are equal, etc). Thus the light we observe necessarily must have been scattered by planes on the surface normal to the vector that bisects the phase angle. Even on unusual surfaces, microroughness will provide the appropriate scattering facets. In the extreme argument, should the plane be a perfectly flat surface (e.g. a mirror) it will scatter no light to the observer when away from a perfect alignment. This will decrease the overall flux but not change the percent of the received flux polarized by the surrounding area. If aligned perfectly, the plane will still behave polarimetrically as the underlying material it is made of, polarizing the same fraction of the light as determined by its albedo. Thus 
even in case of extreme topography, percent polarization measurements will only be sensitive to the underlying material composition.

Changes in the absolute level of polarization as the phase angle changes nightly precludes wrapping all four nights of observing onto a single rotation phase. However we can correlate features at similar rotation phases across nights and from this fit different polarization slopes to different locations on the asteroid. We use the phase range of $0.6-0.8$ to represent the peak polarization and the phase range of $0.8-0.4$ (wrapped) to represent the baseline background polarization (as determined from the 2009-07-22 observing night). We interpret the phase range of $0.4-0.6$ as a transition region (see below) and do not include it in either measurement. Using the summed maximum and baseline values for the first night as location benchmarks we determine the absolute change in albedo across the surface of Anteros.

We find a background surface albedo of $p_{v}=0.181 \pm 0.002 \pm 0.02$ with a single spot of much lower albedo. Note that this value does not vary significantly from the albedo of $p_{v}=0.13 \pm 0.03$ found from radiometric modeling (Veeder, et al. 1981) or the one published in the compilation by Chapman, et al. (1994) of 0.17 (no error given). We measure an upper limit to the albedo for the dark area of $p_{v}=0.160 \pm 0.004 \pm 0.02$ however this feature is unresolved and thus the albedo measurement assumes coverage of the maximal possible area allowable by the observing geometry ( $44 \%$ of the total surface area for 2009-07-22, corresponding to a projected area of $3.7 \mathrm{~km}^{2}$ ). It is likely that the dark spot covers a much smaller area with a much lower reflectance.

Reflected polarization from a mottled surface will mix when unresolved to give a value between the two extremes. For the case of Anteros on the night of 2009-07-22, taking a background polarization for $\alpha=40.5^{\circ}$ of $P_{b k g}=2.46 \%$ (the mean of the baseline polarization value for the night) and a peak measured polarization of $2.70 \%$ we find that the true percent polarization of the dark spot $\left(P_{d a r k}\right)$ on that night can be described as

$$
P_{d a r k} * C+P_{b k g}(1-C)=2.7
$$

where $C$ is the fraction of the projected illuminated surface that the dark region covers. This is simply because the polarized light from the dark area is diluted by the signal from any background material also visible. This relation can be simplified to:

$$
P_{\text {dark }}=\frac{0.24}{C}+2.46
$$

The 2009-07-22 data plotted in Fig 2 show a gradual build up in the polarization value with a rapid falloff from the peak level to the base level over slightly more than one tenth of a rotation. This is likely due to the dark region rising over the horizon as seen from Earth and then passing from the lit side quickly across the terminator and thus out of illumination. This scenario would require Anteros to have a prograde rotation state. From the rapid falloff we can calculate that the maximum size of the dark feature along the direction of rotation is $<0.7 \mathrm{~km}$ assuming it is located on the equator of the asteroid. If the spot is not on the equator this argument would derive a smaller value for the size. 
Using this size as the diameter of a circular crater this corresponds to a total projected surface coverage of the dark spot of $C<11 \%$ (equivalent to $<2 \%$ of the total unprojected surface area, assuming the rotation pole is parallel to the plane of the sky), giving a polarization value for this area of $P_{\text {dark }}=4.7 \%$. If instead the rotation axis is inclined to the plane of the sky, the projected area would be smaller. This would increase the required value of $P_{\text {dark }}$ which would cause the calculated value for the albedo (see below) to decrease. Thus the assumptions made here represent the 'brightest-case' scenario for the spot.

As the polarization for the other three nights is consistent at all phases we can only set an upper limit for the value of $P_{d a r k}$ on those nights based on a maximum unresolved change in percent polarization of $0.1 \%$. Using the analouges of Eq 3 for the other nights we find limits of $P_{\text {dark }}<1.85 \%$ for the night of 2009-08-11, $P_{\text {dark }}<0.88 \%$ for 2009-08-26, and $P_{\text {dark }}<-0.14 \%$ for 2009-09-10. We show the polarization for the dark region separated out from the background material in Fig 3 as well as the same S- and C-type generic models from Fig 1, Using these values we calculate a lower limit on the slope and thus an upper limit on albedo. The albedo of the dark spot is limited to $p_{v}<0.09$.

\section{Conclusions}

Using DBIP on the University of Hawaii's $2.2 \mathrm{~m}$ telescope we have investigated the NEA (1943) Anteros for surface heterogeneity. We find that Anteros shows significant polarimetric variation as a function of rotation at high phase angles, implying an albedo gradient and corresponding surface composition variations. We determine that Anteros has a base albedo of $p_{v}=0.181 \pm 0.002 \pm 0.02$ (errors are relative and absolute, respectively) consistent with literature values as well as a dark spot of albedo $p_{v}<0.09$ covering $<2 \%$ of its surface.

A single small asteroid showing albedo variations does not invalidate the assumption that shape dominates the light curves of these bodies. Indeed it is clear from spacecraft visits that shape does play an important role in determining the reflected flux. Additionally, albedo variations for most asteroids appear to be small and localized. However the potential for albedo variations for small asteroids can not be discounted outright.

It is currently unclear what processes could cause localized albedo changes across the surfaces of small asteroids. Further polarimetric studies of small NEAs are thus necessary to constrain the frequency of albedo and composition changes across the surfaces of these bodies. Once an account the population is established evolutionary pathways to the creation of these features can be explored. 


\section{Acknowledgments}

J.M. was supported under NASA PAST grant NNG06GI46G. The author would like to thank Rob Jedicke and Alan Tokunaga for providing comments on the manuscript, as well as V. Rosenbush and an anonymous referee for helpful reviews that improved the paper. The author wishes to recognize and acknowledge the very significant cultural role and reverence that the summit on Mauna Kea has always had within the indigenous Hawaiian community. I am most fortunate to have the opportunity to conduct observations from this sacred mountain. 


\section{REFERENCES}

Akimov, L.A., Lupishko, D.F. \& Belskaya, I.N., 1983, "Photometric heterogeneity of asteroid surfaces", Soviet Astronomy, 27, 577.

Binzel, R.P., Birlan, M., Bus, S.J., Harris, A.W., Rivkin, A.S. \& Fornasier, S., 2004, "Spectral observations for near-Earth objects including potential target 4660 Nereus : Results from Meudon remote observations at the NASA Infrared Telescope Facility (IRTF)", P\&SS, 52, 291.

Broglia, P. \& Manara, A., 1992, "A study of the polarimetric lightcurve of the asteroid 16 Psyche", A\&A, 257, 770.

Broglia, P. \& Manara, A., 1989, "Rotational variations in the optical polarization of 4 Vesta", A\&A, 214, 389.

Cellino, A., Gil Hutton, R., di Martino, M., Bendjoya, Ph., Belskaya, I.N. \& Tedesco, E.F., 2005, "Asteroid polarimetric observations using the Torino UBVRI photopolarimeter", Icarus, 179, 304.

Cellino, A., Gil Hutton, R., Tedesco, E.F., di Martino, M. \& Brunini, A., 1999, "Polarimetric Observations of Small Asteroids: Preliminary Results", Icarus, 138, 129.

Cellino, A., Zappala, V., di Martino, M., Farinella, P. \& Paolicchi, P., 1987, "Flattening, pole, and albedo features of 4 Vesta from photometric data", Icarus, 70, 546.

Chapman, C.R., Harris, A.W. \& Binzel, R., 1994, "Physical properties of near-Earth asteroids: implications for the hazard issue", Hazards Due to Comets and Asteroids, ed. Gehrels (Univ of Arizona Press), 537.

Degewij, J., Tedesco, E.F. \& Zellner, B., 1979, "Albedo and color contrasts on asteroid surfaces", Icarus, 40, 364.

Fossati, L., Bagnulo, S., Mason, E. \& Landi Del'Innocenti, E., 2007, "Standard Stars for Linear Polarization Observed with FORS1", ASP Conf., 364, 503.

Kaasalainen, M., Torppa, J. \& Muinonen, K., 2001, "Optimization Methods for Asteroid Lightcurve Inversion. II. The Complete Inverse Problem", Icarus, 153, 37.

Lupishko, D.F., BelSkaia, I.N., Kvaratskheliia, O.I., Kiselev, N.N. \& Morozhenko, A.V, 1988, "Polarimetry of Vesta in 1986 opposition", Astron.Vestnik, 22, 142.

Masiero, J., Hodapp, K.-W., Harrington, D. \& Lin, H., 2007, "Commissioning of the Dual-Beam Imaging Polarimeter for the UH 88-inch telescope", PASP, 119, 1126. 
Masiero, J., Hodapp, K.-W., Harrington, D. \& Lin, H., 2008, "Extended Commissioning and Calibration of the Dual-Beam Imaging Polarimeter", to appear in ASP Conf. Series for Astronomical Polarimetry 2008; arXiv:0809.4313.

Masiero, J., Jedicke, R., Ďurech, J., Gwyn, S., Denneau, L. \& Larsen, J., 2009a, "The Thousand Asteroid Light Curve Survey", Icarus, 204, 145.

Masiero, J., Hartzell, C. \& Scheeres, D.J., 2009b, "The effect of the dust size distribution on asteroid polarization", AJ, 138, 1557.

Morbidelli, A., Bottke, W., Nesvorný, D. \& Levison, H.F., 2009, "Asteroids were born big", Icarus, in press.

Mitchell, D.L., Ostro, S.J., Rosema, K.D., Hudson, R.S., Campbell, D.B., Chandler, J.F. \& Shapiro, I.I., 1995, "Radar observations of asteroids 7 Iris, 9 Metis, 12 Victoria, 216 Kleopatra, and 654 Zelinda", Icarus, 118, 105.

Muinonen, K., 1989, "Electromagnetic Scattering by Two Interacting Dipoles", Proc. URSI International Symp. on Electromagnetic Theory, 428.

Muinonen, K., Piironen, J., Kaasalainen, S. \& Cellino, A., 2002a, "Asteroid photometric and polarimetric phase curves: empirical modeling", Mem. S.A.It., 2002, 73, 716.

Muinonen, K., Piironen, J., Shkuratov, Y., Ovcharenko, A. \& Clark, B., 2002b, "Asteroid Photometric and Polarimetric Phase Effects", Asteroids III, ed. Bottke, Cellino, Paolicchi \& Binzel (Univ of Arizona Press), 123.

Muinonen, K., Videen, G., Zubko, E. \& Shkuratov, Yu., 2002b, "Numerical Techniques for Backscattering by Random Media", Optics of Cosmic Dust, ed. Videen \& Kocifaj (NATO Science Series), 79, 261.

Nakayama, H., Fujii, Y., Ishiguro, M., et al., 2000, "Observations of Polarization and Brightness Variations with the Rotation for Asteroids 9 Metis, 52 Europa, and 1036 Ganymed", Icarus, $146,220$.

Pravec, P., Harris, A. W., Michaĺowski, T., 2002, "Asteroid Rotations", Asteroids III (W. F. Bottke, Jr. et al., eds.), 113.

Pravec, P., Wolf, M. \& Sarounova, L., 1998, "Lightcurves of 26 Near-Earth Asteroids", Icarus, 136, 124.

Schmidt, G.D., Elston, R., \& Lupie, O.L., 1992, "The Hubble Space Telescope Northern-Hemisphere Grid of Stellar Polarimetric Standards", AJ, 104, 1563.

Thomas, P.C., Binzel, R.P., Gaffey, M.J., Storrs, A.D., Wells, E.N. \& Zellner, B.H., 1997, "Impact excavation on asteroid 4 Vesta: Hubble Space Telescope results", Science, 277, 1492. 
Veeder, G.J., Tedesco, E.F., Tholen, D.J., Tokunaga, A., Kowal, C.T., Matthews, K., Neugebauer, G. \& Soifer, B. T., 1981, "The diameter and albedo of 1943 Anteros", Icarus, 46, 281.

Zellner, B., Gehrels, T., \& Gradie, J., 1974, "Minor planets and related objects. XVI. Polarimetric diameters", AJ, 79, 1100.

This preprint was prepared with the AAS $\mathrm{LAT}_{\mathrm{E}} \mathrm{X}$ macros v5.2. 
Table 1: Polarimetry of (1943) Anteros

\begin{tabular}{cccccccc}
\hline UT Obs Date & V mag & $t_{\text {exp }}(\mathrm{sec})$ & $n_{\text {meas }}$ & $\alpha$ & Ecliptic Long & Lin \% Pol & $\theta_{p}$ \\
\hline $2009-07-22$ & 16.6 & 130 & 21 & $40.5^{\circ}$ & $356.9^{\circ}$ & $2.53 \pm 0.02$ & $178.8 \pm 0.3^{\circ}$ \\
$2009-08-11$ & 16.3 & 130 & 26 & $28.0^{\circ}$ & $352.9^{\circ}$ & $0.83 \pm 0.02$ & $0.8 \pm 0.6^{\circ}$ \\
$2009-08-26$ & 16.1 & 90 & 27 & $19.1^{\circ}$ & $346.3^{\circ}$ & $-0.14 \pm 0.02$ & $86 \pm 4^{\circ}$ \\
$2009-09-10$ & 16.3 & 100 & 30 & $16.4^{\circ}$ & $339.3^{\circ}$ & $-0.41 \pm 0.02$ & $94 \pm 1^{\circ}$ \\
\hline
\end{tabular}




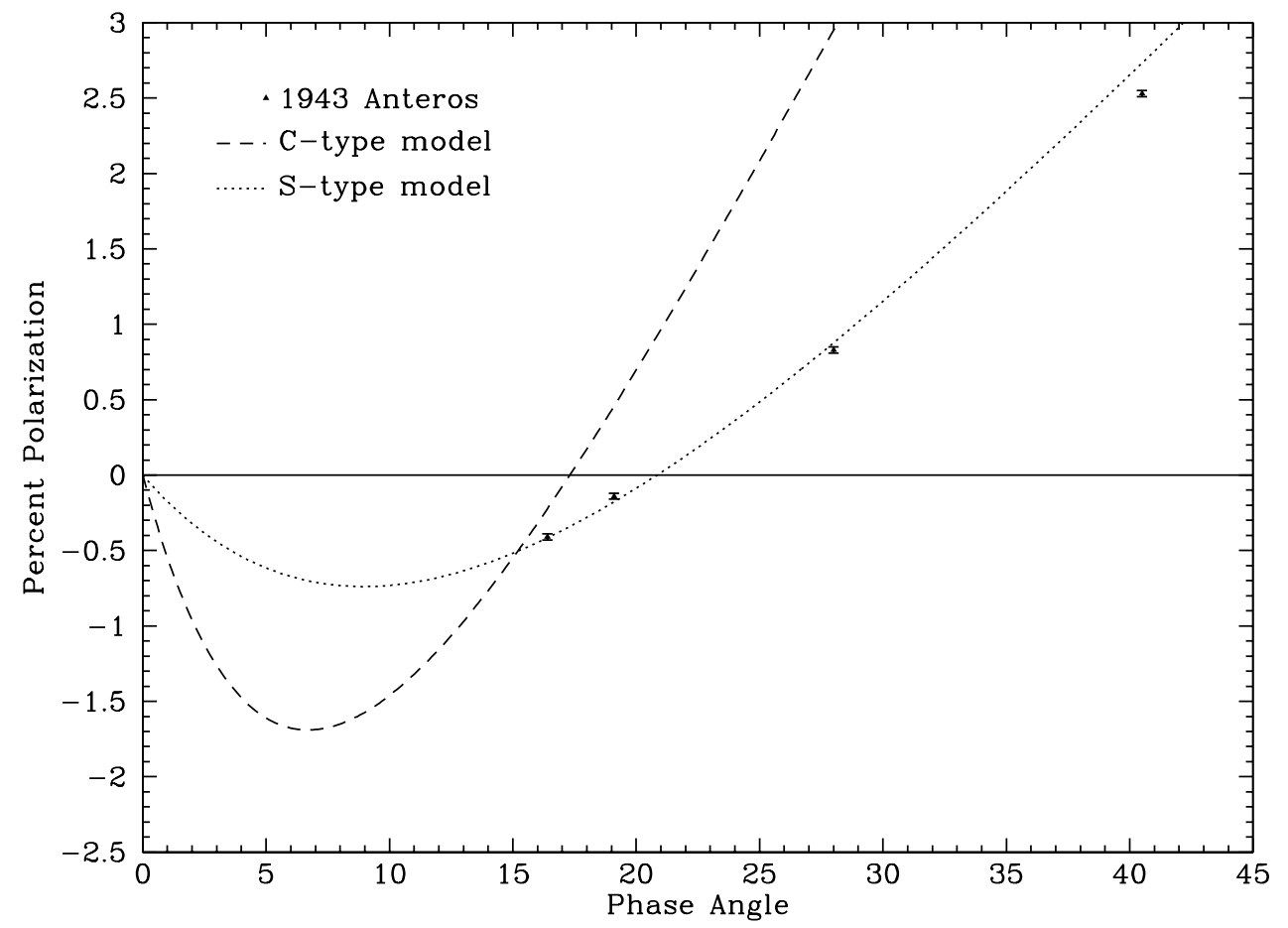

Fig. 1.- New observations of the polarization of (1943) Anteros along with model curves for S-type (dotted) and C-type (dashed), for reference. Note the errors on the percent polarization are comparable to the size of the points. 


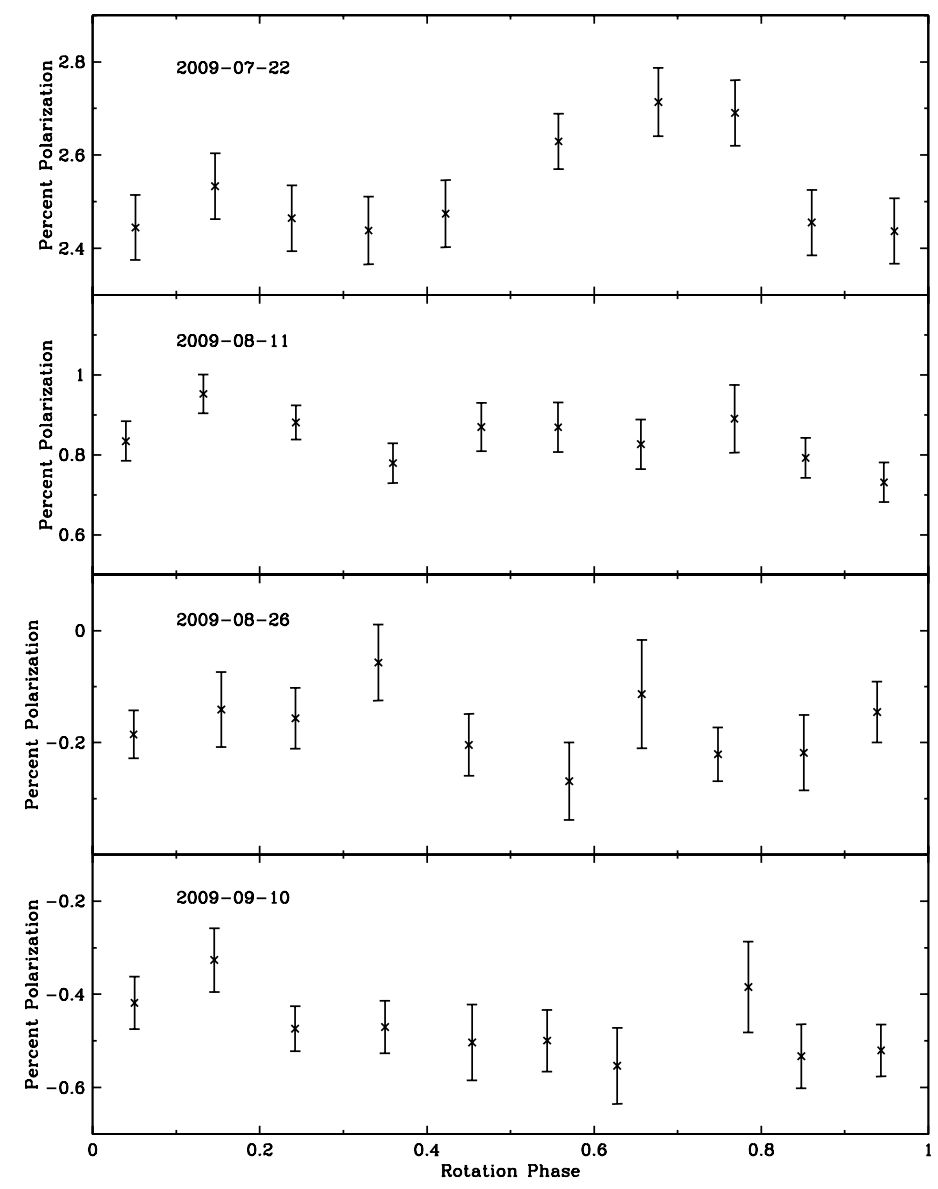

Fig. 2.- Polarization of Anteros as a function of rotation phase. Observations within 0.1 phasewide bins have been co-added to reduce errors. UT date of each observation set is listed. 


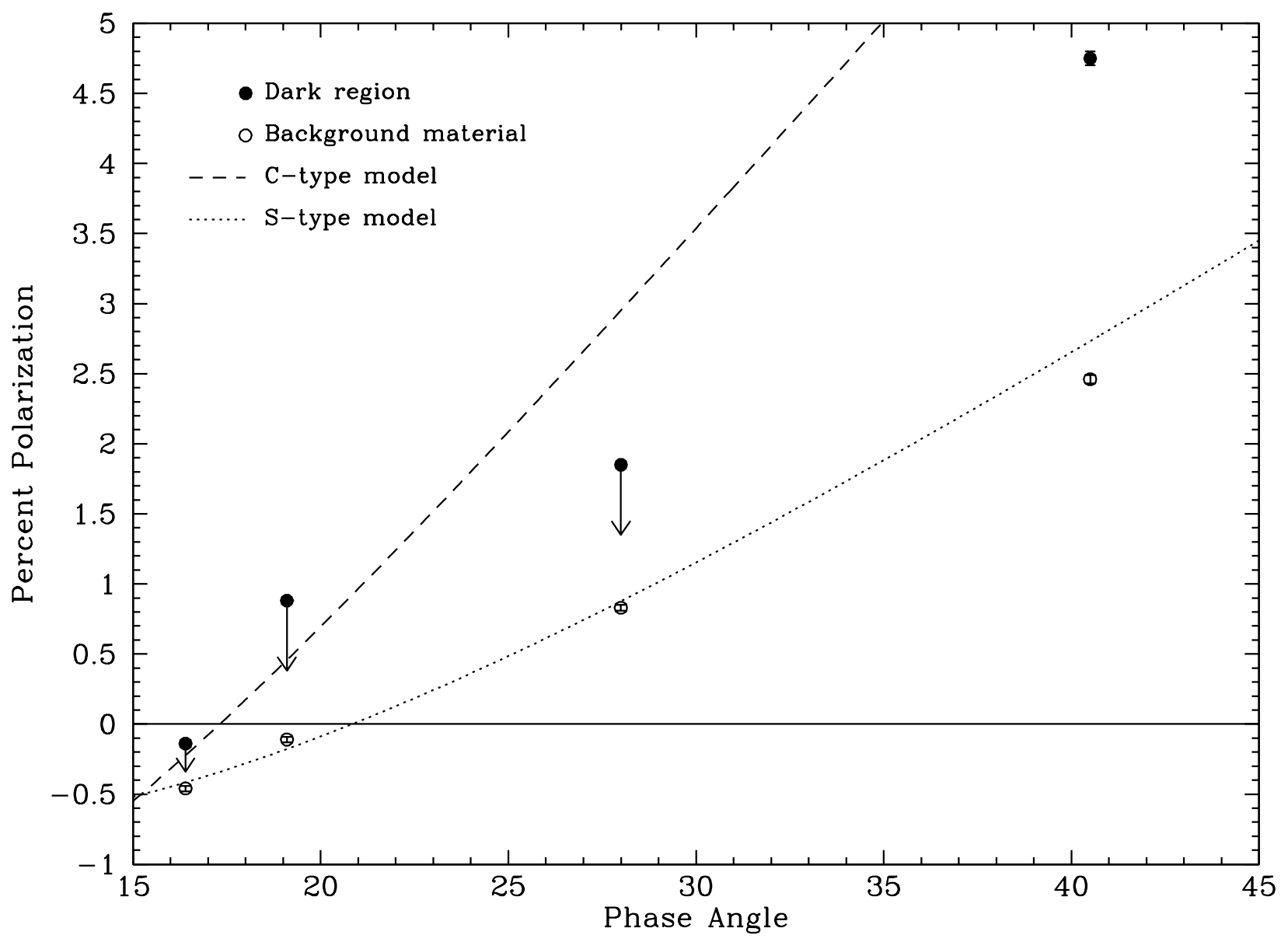

Fig. 3.- Polarization of the background and the dark region of (1943) Anteros. The values of the polarization for the dark region for phase angles below $40.5^{\circ}$ are upper limits. Note that the error bars on the points are comparable to their size. 